A N N A L E S Annales de Bretagne et des Pays de l'Ouest

Anjou. Maine. Poitou-Charente. Touraine

111-3 | 2004

Alcuin de York à Tours

\title{
Alkuin und das Quadrivium in der Karolingerzeit
}

\section{Brigitte Englisch}

\section{(2) OpenEdition}

Journals

Édition électronique

URL : http://journals.openedition.org/abpo/1233

DOI : $10.4000 / a b p o .1233$

ISBN : 978-2-7535-1495-9

ISSN : 2108-6443

Éditeur

Presses universitaires de Rennes

Édition imprimée

Date de publication : 20 septembre 2004

Pagination : 163-174

ISBN : 978-2-7535-0053-2

ISSN : 0399-0826

Référence électronique

Brigitte Englisch, «Alkuin und das Quadrivium in der Karolingerzeit », Annales de Bretagne et des Pays de I'Ouest [En ligne], 111-3| 2004, mis en ligne le 20 septembre 2006, consulté le 19 avril 2019. URL

http://journals.openedition.org/abpo/1233 ; DOI : 10.4000/abpo.1233 


\title{
Alkuin und das Quadrivium in der Karolingerzeit
}

\author{
Brigitte ENGLISCH \\ Professeur d'histoire médiévale à l'Université de Paderborn
}

Alkuins bekanntes Werk zum Quadrivium sind die ihm zugeschriebenen Propositiones ad acuendos juvenes (= Aufgaben zur Schärfung des Geistes der Jungend) ${ }^{1}$, im folgenden als Propositiones zitiert, die zu den vielleicht wichtigsten Sammlungen von Rechen- und Scherzaufgaben in lateinischer Sprache gehören. Die Faszination dieses in der ältesten Handschrift (Vat. Regin. Lat. 309, f. $16^{\text {rv }}$, f. $3^{\mathrm{v}}-4^{\mathrm{r}}$ ) aus dem späten 9. Jh. überlieferten Werkes ${ }^{2}$ beruht weitgehend auf der Tatsache, dass wir hiermit eines der wenigen Rechenbücher des Frühmittelalters vor uns haben, dessen Nutzung im Kreis der Hofgesellschaft um Karl den Grossen angenommen werden kann. Daher ist es naheliegend, dass mit den arithmetischen Scherzfragen für Einhard, den "figurae arithmeticae subtilitatis«, von denen Alkuin in einem Brief an Karl den Großen aus dem Jahr 800 (Alc. Ep. 172) spricht, die Propositiones gemeint sind. Zudem würde der unterhaltsame Charakter der Propositiones durchaus zu den Gesprächen zwischen den Gelehrten am Hof passen, an denen bisweilen auch Karl selbst teilnahm; so scheint es vorstellbar, dass es die in den Propositiones vorgeführten Aufgaben waren, die die im abendlichen Kreis um "David" und "Flaccus" versammelte Hofgesellschaft mit der Suche nach einer Lösung unterhielten ${ }^{3}$ und die dann vom virum doctissimum, wie Einhard ${ }^{4}$ Alkuin mit Ehrerbietung nennt, erläutert wurden.

1. Die maßgebliche Edition des Textes stammt von M. FoLKERTS, « Die älteste mathematische Aufgabensammlung... ", der damit die alte Migne-Ausgabe ersetzte. Die kritische Textausgabe wurde nochmals abgedruckt und mit einer Übersetzung versehen in der Ausgabe von M. FOLKERTS und H. GERICKE, "Die Alkuin zugeschriebenen Propositiones... ».

2. Wahrscheinlicher Entstehungsort ist das Kloster St. Denis bei Paris; zur Überlieferungsproblematik s. M. FOLKERTS, « Die älteste mathematische Aufgabensammlung... », S. 17-23.

3. S. hierzu D. Hagermann, Karl der Grosse..., S. 656.

4. Einhard, Vita Karoli magni, cap. 25: [...] in ceteris disciplinis Albinum cognomento Alcoinum, item diaconum, de Brittania Saxonici generis hominem, virum undecumque doctissimum, praeceptorem habuit, apud quem et rhetoricae et dialecticae, praecipue tamen astronomiae ediscendae plurimm et temporis et laboris inpertivit. Discebat artem conputandi et intentione sagaci siderum cursum curiosissime rimabatur. 
Dennoch hat gerade diese Vorstellung wie auch der von Menso Folkerts dieser Aufgabensammlung beigelegte Genrebegriff der "Unterhaltungsmathematik ${ }^{5}$ « dazu beigetragen, dass sie bis jetzt noch nie im Hinblick auf ihre Bedeutung für das frühmittelalterliche Rechnen und auf ihren Stellenwert im Hinblick auf die Probleme der operativen Arithmetik der Epoche hin untersucht worden sind ${ }^{6}$. Es stellt sich mithin die Frage, und dies soll im Mittelpunkt meines Vortrages stehen, ob die in den Propositiones vorgeführten Aufgaben wirklich allein den Zweck einer intellektuellen Gedankenspielerei besaßen, oder ob hier nicht Methoden geübt und Probleme erläutert wurden, die im Zusammenhang mit dem praktischen Rechnen der Epoche, vornehmlich im Umfeld der Komputistik stehen.

Genau in diesem Punkt manifestieren sich im Frühmittelalter einige erhebliche Schwierigkeiten, die auf das mathematische Vermögen der Epoche zurückzuführen sind, insbesondere das System der römischen Zahlen, die auch im Mittelalter ihre Geltung bewahrten. Diese bilden, im Unterschied zu unseren heutigen Dezimalzahlen kein Stellenwertsystem, d. h. ein System, bei dem die Position beispielsweise der Ziffer 3 ausschlaggebend dafür ist, ob es sich um den Wert 3, 30, 300 oder 3000 handelt. Die römischen Zahlen bilden vielmehr ein additives Ziffernsystem ${ }^{7}$.

Damit wird die operative Arithmetik der römischen Antike und des Frühmittelalters durch ein komplexes Zahlensystem geprägt, wie es sich anhand der mittelalterlichen Multiplikationstabellen ${ }^{8}$ leicht verdeutlichen lässt. Man rechnet zunächst einmal nicht mit einheitlichen Ziffern, sondern einem System, welches einen Strich- und Buchstabencode den Zahlenwerten $1,5,10,50,100,500,1000$ und deren Vielfachen zuordnet. Alle weiteren Zahlen werden durch Kombination, d. h. Aneinanderreihung dieser Basiswerte gebildet, wobei jedoch nicht ihre Position ausschlaggebend ist. Entscheidend sind allein die einzelnen Nominalwerte, die zu addieren sind. Hieraus resultieren für das operative Rechnen der Epoche gleich mehrere Effekte: zunächst einmal können Zahlenwerte nicht "auf den ersten Blick" erfasst werden. Man ist vielmehr gezwungen, schon zur Bestimmung einer Zahl zu rechnen, wie das Beispiel der Zahl MMDCCCCLX zeigt, bei der wir zunächst festzustellen haben, dass wir zwei Tausender, einen Fünfhunderter, vier Hunderter, einen fünfziger und einen Zehner haben, die wir zur Zahl 2960 addieren müssen. Ist bereits die Erfassung einer Zahl ein solches Problem, kann leicht verdeutlicht werden, das die Bewältigung konkreter Aufgaben in diesem Zusammenhang von massiven Schwierigkeiten gekenn-

5. M. FOLKERTS, " Die älteste mathematische Aufgabensammlung... », S. 35 ; M. FOLKERTS und H. GERICKE, " Die Alkuin zugeschriebenen Propositiones... ", S. 284.

6. So befasst sich auch die Studie von D. SingMASTER, "The History... ", vornehmlich mit der möglichen Deszendenz und der Entschlüsselung mancher Aufgaben mit modernen mathematischen Methoden.

7. Zum römischen Zahlensystem s. z. B. K. MENNINGER, Zahlwort und Ziffer...

8. Ein sehr ansprechendes Beispiel stellt die Multiplikationstabelle aus einer komputistischen Handschrift aus dem 10. Jh. (Angers, Bibliothèque Municipale, ms. 477, $\mathrm{f}^{\circ} 47 \mathrm{v}^{\circ}$ ) dar; s. hierzu J. Dalarun, Le Moyen Âge en lumière, S. 41, fig. 8. 
zeichnet war. Zwar sind Addition und Subtraktion aufgrund dieser Struktur noch relativ leicht zu bewerkstelligen; jedoch ist eine Multiplikation nur mit Umwegen realisierbar und eine Division mit variablen Zahlenwerten höchst umständlich. Konform dazu ist auch die mit den Aufgaben des Teilens unabdingbar verknüpfte Technik einer Bruchrechnung im frühen Mittelalter nur unter Zuhilfenahme von Ergebnistabellen, den sog. Calculi ${ }^{9}$ möglich, die gelegentlich auch als Rechenknechte bezeichnet werden. Sie dienten dazu, die Resultate bezüglich solcher Zahlenverhältnisse einfach abzulesen.

Stellen wir folglich die Frage, wie man in dieser Epoche nun tatsächlich gerechnet hat und wie man mit diesen Schwierigkeiten umgegangen ist, so geben die relevanten Quellen, z. B. die Bildungsschriften zum Quadrivium, d. h. zur Arithmetik, Geometrie, Astronomie und Musik, nur wenig konkrete Hinweise. Dies ist im wesentlichen auf die Art des dort vorgeführten Wissens zurückzuführen. Konsultieren wir in diesem Zusammenhang die repräsentative Enzyklopädie des Mittelalters, die Etymologiae Isidors von Sevilla ${ }^{10}$, kann unschwer verdeutlicht werden, worin das Dilemma frühmittelalterlicher Mathematik bestand: ihrem Charakter einer praxisfernen Formelgelehrsamkeit ${ }^{11}$. Zwar galt bei Isidor wie bei den anderen frühmittelalterlichen Enzyklopädisten die Arithmetik als zentrale Disziplin, da sie zum Verständnis aller anderen unabdingbar sei ${ }^{12}$, doch war seine Zahlenlehre alles andere als eine Anleitung zum konkreten Rechnen. In der Nachfolge spätantiker Lehrschriften ${ }^{13}$ konzentrieren sich seine Ausführungen auf das Wesen der Zahl und dem Verhältnis der Zahlen zueinander. So beschäftigt er sich zunächst mit den Eigenschaften und Namen der Zahlen von 1-5, 6, 10, 10-30, 100-300 und widmet sich dann den althergebrachten Wissensätzen zu den Gruppen der geraden und ungeraden Zahlen, der Teiler gerader und ungerader Zahlen und der Zahlenproportionen. So gilt ihm, um ein Beispiel zu präsentieren, als superparticularis numerus, diejenige größere Zahl, die eine kleinere zuzüglich eines weiteren Teils in sich einschließt, wie die 3 , die die zwei und die eins enthält, wobei die eins die Hälfte von zwei sei ${ }^{14}$. Eine subsuperparticularis numerus hingegen sei eine kleinere Zahl, die eine größere zuzüglich eines Teils von dieser enthalte, wie 2 zu 3, 3 zu 4, 4 zu 5 usw $^{15}$.

9. Die aktuellste Edition des Calculus des Victorius und des Abbo von Fleury findet sich bei A. M. PEDEN, Abbo of Fleury...

10. Isidor von Sevilla, Etimologias; $\mathbf{s}$. hierzu bes. J. FonTAINE, Isidore de Séville et la culture classique...

11. B. Englisch, Die Artes liberales..., S.127 ff.; J. FontAINE, « Isidore de Séville et la mutation... ", S. 43 ff. sowie P. Riche, De l'éducation antique...

12. Isidor von Sevilla, Etymologiae 3,1 (1, S. 422).

13. B. Englisch, Die Artes liberales, bes. S. 132-136.

14. Isidor von Sevilla, Etymologiae 3, 7 (1, S. 430) : Superparticularis numerus est, dum fortior continet intra se inferiorem numerum, circa quem conparatur, similiter et unam partem eius; ut verbi gratia III ad II dum conparati fuerint, continent intra se II et alium unum, qui media pars est duorum; [...].

15. Isidor von Sevilla, Etymologiae 3, 10 (1, S. 430) : Subsuperparticularis numerus est minor, qui continetur in fortiori numero cum alia una parte sua, aut media, aut tertia, aut quarta, aut quinta; ut verbi II ad III, III ad IV, IV ad V, et ceteri. 
Bereits dieser kurze Exkurs macht deutlich, dass hier nicht das Erlernen praktischen Rechnens im Vordergrund stand, sondern eine an antike Bildungsprämissen anknüpfende Lehre über die Wesenheit der Zahlen, die in elementare, memorierbare Regeln gebracht worden war. Dennoch sollte bei der Bewertung der klassischen Gelehrsamkeit in diesem Quadriviumsfach nicht allein das statisch, realitätsabgewandte Element hervorgehoben werden. Die vielen minutiösen Definitionen, die Isidor hier vorgibt, können dabei auch als eine Reaktion auf die Hindernisse des römischen Zahlensystems bewertet werden. In einem im Grunde für komplexe Operationen nicht ausgelegten Zahlensystem mag es durchaus sinnvoll erschienen sein, Definitionen vielfältigster Art vorzugeben, gewissermaßen als Hilfestellung beim Umgang mit dieser komplexen Materie. Das Ergebnis all dieser Bemühungen wird aber in keinem Fall darin bestanden haben, Fähigkeiten im profanen Bereich praktischen Rechnens hervorzubringen.

Gerade für Alkuin dürfte sich das Problem gestellt haben, dass die elitärabstrakten Lehren der Handbucharithmetik und ihren vorgefertigten Standardgrößen nicht ausreichten, um praktisch/arithmetische Aufgabenstellungen zu bewältigen. Die Beschäftigung mit den Fragen der Zahlenlehre, aber auch der angewandten Mathematik war im Frühmittelalter nämlich nicht nur Bestandteil des Quadriviums, sondern - neben den notwendigen astronomischen Kenntnissen - elementare Voraussetzung für die Osterfestberechnung. So setzte der für das Mittelalter bedeutsame Themenkomplex der Komputistik - immerhin galt gemäß Beda Venerabilis im frühen 8. Jh. ein Bruch mit der Osteregel des Nicäums als Anzeichen der manichäischen Häresie ${ }^{16}$ - die langfristige zyklische Vereinigung von Sonnenjahr und Mondmonaten und mithin der Rechengrößen 365 1/4, 29 1/2 und 27 1/3 voraus. Dies zog eine Reihe offenkundig komplexer Teilungsoperationen nach sich. Es bestand in diesem Bereich also die unumgängliche Notwendigkeit, differenzierte und komplexe Berechnungen vorzunehmen.

Diesem Gebiet galt ein zentrales Interesse Karls des Grossen, vornehmlich im Hinblick auf die Vereinheitlichung der Osterfestberechnung, die Mitte des 8 . Jh. noch nicht vollzogen gewesen zu sein scheint. Zumindest wird von Fredegar für das Jahr 742 von einem Osterstreit berichtet ${ }^{17}$, der vermutlich

16. Beda Venerabilis, De temproum ratione, dans Charles W. JonEs (ed.), Bedae opera de temporibus, S. 175-291, hier cap. 59, S. 279/280: Atque insuper in huius poenam peccati vicesimum secundum diem, qui in tota paschali institutione per Moysen nec semel appellatus invenitur, frequenter in sui paschae principium sanciri praecipiunt. [...] deinde ne dominica die luna quarta decima constituta ieiunare cogamur - indecentem rem illicitamque facientes, hoc enim manichaeorum sectae consuetudo possedit. Diese Edition kann aufgrund ihres unverzichtbaren kritischen Kommentars durch die Neuausgabe des Werkes Beda Venerabilis, De temporum ratione, ed. Charles W. JonEs, dans CCSL 123 B (Bedae Venerabilis opera. Pars 6, Opera didascalia 2), S. 262-460 lediglich ergänzt, nicht aber ersetzt werden, aus welchem Grund auch hier nach ersterer zitiert wird. Zu diesem Werk s. zuletzt B. ENGLISCH, Die Artes liberales, S. 280-395 sowie F. WALLIS, Bede : The Reckoning of Time, Liverpool 1999.

17. Fredegarius Scholasticus, Chronica libri IV cum continuationibus, S. 1-193, hier cap. 24, S. 179 : Interim, quod dici dolor et meror est, sollicitatur ruina, in sole et luna et stellis nova 
auf ein paradoxes Datum nach dem in Gallien gebräuchlichen Zyklus des Victorius von Aquitanien zurückzuführen ist. Karl der Grosse scheint, konform zu seinen anderen Bestrebungen, sein Reich zu ordnen ${ }^{18}$, auch hier darauf bedacht gewesen zu sein, eindeutige Normierungen vorzunehmen; hierauf deutet zumindest sein Bemühen hin, den vermutlich im letzten Drittel des 8. Jh. in St. Médard de Soissons entwickelten Kalender ${ }^{19}$ wie auch die Schriften des Beda Venerabilis zur Komputistik in seinem Reichsgebiet zu verbreiten ${ }^{20}$. Auch die Admonitio generalis aus dem Jahr 789, in der er die Anweisung erlässt, jeder Kleriker müsse die Komputistik beherrschen ${ }^{21}$, zeugt von der Nachhaltigkeit, die Einheit und Funktionalität seiner Herrschaft auch in seiner Beherrschung der Parameter der Zeit zu demonstrieren. Dieses setzt aber voraus, das man bestimmte, keineswegs unerhebliche Rechenoperationen durchführen musste, selbst wenn man eine Ostertafel benutzte. Dies dürfte vor allem Alkuin betroffen haben, den zuletzt Kerstin Springsfeld ${ }^{22}$ als spiritus rector bei der Umsetzung dieser Maßnahmen hervorgehoben hat, indem sie seine Autorschaft für das komputistische Werk Ratio de luna ${ }^{23}$ nachwies und für die Calculatio Albini Magistri $^{24}$ als zumindest vorstellbar charakterisierte. Die damit verbundenen Aufgabenstellungen und Methoden waren in diesem Zusammenhang nicht mehr profane Praxis, sondern ein theologisches wie politisches Desiderat.

Eine Schrift, wie die Propositiones Alkuins, die sich mit der Lösung konkreter Rechenprobleme beschäftigt, gibt daher gerade aufgrund ihrer Einzigartigkeit einen einmaligen Einblick in den epochenspezifischen Umgang mit den Problemen konkreten Rechnens. Insgesamt gesehen präsentieren sich die Propositiones in Gestalt von Textaufgaben, d. h. die eigentliche Rechnung ist in eine Erzählung eingekleidet. Die Aufgabenstellung wird in Form einer an einen Zuhörer oder Leser gestellten Frage formuliert; direkt im Anschluss daran findet sich die Solutio die die Überlegungen und Gedankenschritte erläutert, die zur Lösung führen, jedoch zumeist ohne dezidierte Angabe des Lösungsweges. Dabei ist die Themenstellung auf den ersten Blick ausgesprochen different; es finden sich Aufgaben aus dem klösterlichen Bereich, bei denen es um die Verteilung von Broten unter den Mönchen geht, aus der ländlichen Lebenswelt, die den Verkauf von Schweinen oder Getreidemaße betreffen, oder aus dem Handwerk, wie die Herstellung von Karren. Neben einigen Spezialaufgaben, wie

signa apparuerunt, seu et paschales ordo sacratissimus turbatus fuit. S. hierzu B. ENGLISCH : Zeiterfassung und Kalenderprogrammatik..., S. 82. ff.

18. D. HaGERMAnN, Karl der Grosse..., S. 285-287 und S. $344 \mathrm{ff}$.

19. B. ENGLISCH, Zeiterfassung und Kalenderprogrammatik..., S. 80 ff.

20. B. Englisch, Zeiterfassung und Kalenderprogrammatik..., S. 92 ff. ; D. HaGERMANn, Karl der Grosse..., S. 548.

21. Admonitio generalis, S. 52-62, bes. cap. 72, S. 60. ; s. dazu auch J. FlECKENSTEIN, Die Bildungsreform Karls des Grossen..., S. 24 ff., S. 49-54 und S. 75-85.

22. K. Springsfeld, Alkuins Einfluß..., bes. S. 62 ff und S. 291-294.

23. Alkuin, Ratio de luna, PL 101, Sp. 981C-984B.

24. Alkuin, Calculatio Albini magistri, PL 101, Sp. 999C-1002C. 
Erbschaftsfragen, und reinen Scherzaufgaben ${ }^{25}$, so z. B. wie viel Spuren ein pflügender Ochse hinterlässt - nämlich keine ${ }^{26}$-, ist dabei ein Aspekt mehr als auffällig: Bei 2/3 der in den Propositiones aufgeführten Aufgaben handelt es sich um solche, die Aspekte des Aufteilens, des Verteilens, der Zerlegung vorgegebener Mengen betreffen. Sie sprechen damit den Komplex an, der im römischen Zahlensystem, wie oben erwähnt, faktisch kaum realisierbar, aber für die Umsetzung der Osterfestrechnung unabdingbar war, nämlich die Division, resp. ihren Umkehrschritt, die Multiplikation.

Dies betrifft aber nicht allein die Art der Rechenoperation, auch ihre Ausgestaltung erinnert an die Methoden, die in der Osterfestrechnung Anwendung fanden. Betrachten wir hierzu die Aufgabe $49^{27}$. Dort wird beschrieben, dass sieben Stellmacher 7 Räder anfertigen. Es wird gefragt, wie viele Wagen sie damit aufrichten können. Als Lösung gibt der Autor an, dass sieben mal sieben 49 ergeben; soviel Räder werden hergestellt. 12 mal vier ergeben 48. Es können also mit 48 Rädern 12 Wagen aufgerichtet werden, wobei ein zusätzliches Rad übrig bleibt. Die Einfachheit der Aufgabe im modernen Zahlensystem sollte dem heutigen Betrachter nicht darüber hinwegtäuschen, dass hier eine im römischen Ziffernsystem komplizierte Aufgabe beinhaltete. So wird die Division 48 durch 12 zu einer Multiplikation umgestaltet. Um zu erfahren, wie oft IIII in XXXXVIIII geht, würde Alkuin also nach der mittelalterlichen Rechenweise zum Ausgangswert 4 immer wieder 4 addieren. D. h. er bildet die Reihe IIII; VIII; XII; XVI; XX; XXIIII; dann stellt er fest, dass die Verdoppelung 48 ergibt. Da er dazu 12 mal die Zahl 4 addieren musste, ist sein Ergebnis 12. Bei Alkuins Vorgehen ist jedoch zudem auffällig, dass er, was hier auch durchaus der konkreten Aufgabenstellung entsprach, keine Bruchrechnung durchführte, sondern einen Rest, gewissermaßen ein Reserverad, zurückbehielt.

Dies ist keineswegs eine Schwäche von Alkuins Rechenmethode, sondern übliche Praxis innerhalb der Komputistik. Angesichts der oben erwähnten Notwendigkeit, einerseits komplexe Teilungen mit nicht ganzzahligem Ergebnis durchzuführen, andererseits der Unzulänglichkeit des Zahlensystems, die eine adäquate Darstellung variabler Brüche nicht ermöglichte, war man auf den genialen Kunstgriff verfallen, die Rechnungen gerade auf die Ermittlung des unteilbaren Restes auszurichten. Diese

25. Zur Beschreibung der einzelnen Aufgaben s. M. FoLKERTs, Die älteste mathematische Aufgabensammlung, S. 34-41.

26. Propositio 14, (ed. M. FolKerTs, S. 52/53) : Bos qui tota die arat, quot vestigia facit in ultima riga? Solutio. Nullum omnino vestigium bos in ultima riga facit, eo quod ipse praecedit aratum et hunc aratum sequitur. Quotquot enim hic praecendo inexculta terra vestigia figit, tot illud subsequens excolendo resolvit. Propterea illius omnino nullum reperitur in ultima riga vestigium.

27. Propositio 49, (ed. M. FolKerTs, S. 73): Septem carpentarii septenas rotas fecerunt. Dicat, qui vult, quot carra erexerunt. Solutio. Duc septies VII, fiunt XLVIIII. Tot rotas fecerunt. XII vero quater ducti XL et VIII reddunt. Super XL et VIIII rotas XII carra sunt erecta, et una superfuit rota. 
Methode ist insofern so einfach, als man zur Lösung einer solchen Aufgabe dann den Teiler nur solange vervielfachen, d. h. durch Addition erweitern muss, bis die so ermittelte Zahl kleiner als der Teiler ist. Das populärste Beispiel ist die Ermittlung der Indiktion, eines häufigen Bestandteils auch der Datierungszeile von Urkunden. Diese errechnet sich gemäß der Formulierung Beda Venerabilis im Kapitel 49 seines komputistischen Hauptwerkes De temporum ratione als Division des um drei erhöhten Inkarnationsjahres durch 15. Für das Jahr 725 bedeute dies, dass man 728 durch 15 dividieren müsse. Da $15 \mathrm{mal} 40$ das Resultat 600 ergebe und $15 \mathrm{mal}$ 8120 , bleibe ein Rest von 8 , welcher folglich als Indiktion zu betrachten sei ${ }^{28}$.

Alkuin trainiert derart also nicht allein ein in der Komputistik übliches Rechenverfahren, auch die von Beda angewandte Methode, eine Teilung in den Umkehrschritt der Division umzugestalten, findet in den Propositiones vielfach Anwendung. Ein schönes Beispiel stellt die Aufgabe 53 dar $^{29}$, in der es darum geht, 204 Eier unter 12 Mönche zu verteilen, die in ihrer Anlage und Rahmenhandlung deutlich an die Statuten Adalhards von Corbie erinnert ${ }^{30}$ und damit eine Verbindung von realem Klosterleben des 9. Jh. und arithmetischen Übungen hervorhebt. Die Antwort beginnt mit der Feststellung, dass man 204 in 12 Teile zerlegen müsse, dabei werde dieser 12. Teil durch den 17. Teil aufgelöst ${ }^{31}$. Interessant ist die nun folgende Begründung, die einen zur Indiktionsberechung analogen Weg aufzeigt: "denn ob du 12 mal 17 oder 17 mal 12 bildest, du findest 204." Auch hier wird also nicht dividiert, sondern multipliziert, d. h. realiter dürfte man so oft 12 addiert haben, bis man das Ergebnis 204 erreichte.

28. Beda Venerabilis, DTR 49 (ed. Ch. Jones, S. 269): Hoc autem argumento quota sit anno quocumque computare volueris indictio reperies : sume annos ab incarnatione domini quotquot fuerint, in praesenti verbi gratia DCCXXV. Adde semper tria, quia quarta indictione secundum Dionysium natus est dominus; fiunt DCCXXVIII. Haec partire per xv (quindecies quadrageni sexcenti, quindecies octoni cenvies); remanent octo. Octava est indictio. Si vero nihil remanserit, quinta decima est.

29. Propositio 53 (ed. M. FolKERTS, S. 75/76): Quidam pater monasterii habuit XII monacjos. Qui vocans dispensatorem domus suae dedit illi ova CCIII iussitque, ut singulis aequalem daret ex eis omnibus portionem. Sic tamen iussit, ut inter V presbyteros daret ova LXXXV et inter quattuor diaconos LXVIII et inter tres lectores LI. Dicat, rogo, qui valet, quot ova unicuique ipsorum in portione evenerunt, ita ut in nullo nec superabundet numerus nec minuatur, sed omnes, ut supra diximus, aequalem in omnibus accipiant portionem. Solutio. Ducentos igitur quattuor per XIIam divide partem. Horum quippe pars XIIa in septima decima resolvitur parte, quia sive duodecies XVII sive decies septies XII miseris, CCIIII reperies. Sicut enim octogenarius quintus numerus septimum decimum quinquefarie de se reddit numerum, ita sexagenarius octavus quadrifarie et quinquagesimus primus trifarie. Iunge ergo V et IIII et III, fiunt XII. Isti sunt homines XII. Rursusque iunge LXXXV et LXVIII et LI, fiunt CCIII. Haec sunt ova CCIIII. Veniunt ergo singulorum es his parte ova XVII perduodeimam partem. Septimum decimum numerum aequa lance divisum.

30. Statuta seu Brevia Adalhardi abbatis Corbeiensis, S. 357-408; s. hierzu B. KASTEN, Adalhard..., bes. S. 110ff.

31. Ich folge hier dem lateinischen Text; zwar entspricht die Form "dieser 12. Teil ist 17 « (M. FOLKERTS und H. GERICKE, Die Alkuin zugeschriebenen Propositiones..., S. 358) besserem Deutsch, die wörtliche Übersetzung macht aber den mühsamen Rechenprozess viel transparenter. 
Das für Alkuin möglicherweise tatsächlich u. a. die Übung von Methoden im Zusammenhang der christlichen Festberechnung eines der Motive bei der Erstellung der Propositiones darstellte, macht auch die erste Aufgabe dieser Sammlung deutlich ${ }^{32}$. Dort schildert Alkuin, dass eine Schnecke von einer Schwalbe zum Essen eingeladen wird, jedoch sei der Weg eine Meile weit. Da die Schnecke nur eine Unze eines Fußes am Tag zurücklegen kann, besteht die Aufgabe darin, zu errechnen, wie viele Tage die Schnecke zum Essen wandert. In der Lösung gibt Alkuin an, dass eine Meile 1500 Doppelschritten und diese wiederum 7500 Fuß entsprechen, was 90.000 Unzen gleichzusetzen sei. Da die Anzahl der Unzen derjenigen der Tage entspreche sei die Schnecke 246 Jahre und 210 Tage unterwegs.

Hier werden gleich mehrere Aspekte der Zeitberechnung angesprochen. So geht es zunächst einmal um eine Umrechnung innerhalb der einzigen der römischen Antike und frühen Mittelalters zur Verfügung stehenden Möglichkeit, Teilungsverhältnisse zu definieren: der sog. Unzenrechnung. Hierbei handelt es sich um die römischen 12er-Brüche, die auf einem Gewichtmaßsystem fußen. Bezeichnenderweise findet sich erneut in Bedas De temporum ratione erstmalig die komplette Präsentation dieses Gewichtsmaßssystems ${ }^{33}$. Es basiert ursprünglich auf der Gleichung 1 As (= Pfund) $=12$ Unzen. So führt Beda die spezifischen Zeichen und Termini für die Teile eines Pfundes libra oder as vor, die die Reihe von 12 Unzen bis hinab zu zwei Unzen umfassen (Abb. $1^{34}$ ). Diese werden gefolgt von der Auflistung der Teile einer Unze, die in 24 Scripuli (oder Scrupel) zerlegt wird. Man darf dieses Gefüge folglich nicht mit einem "Bruchsystem" mit variabeln Zähler und Nenner gleichsetzen, sondern es handelt sich um maßgebundene Zwölferbrüche. Nicht alle Bruchverhältnisse ließen sich folglich in dieser Weise ausdrücken. Dennoch war die Unzenrechnung besonders für die Komputistik dahingehend funktional, als auch viele Zeiteinheiten wie Minuten oder Tagesstunden auf der 12 basierten und auch einfache Zahlenverhältnisse wie ein Viertel, also 3 Unzen, relativ leicht zu erfassen waren. Alkuins Aufgabe rekurriert also auf eine Methode, die gerade für die Komputistik von erhöhter Bedeutung gewesen sein dürfte.

In Aufgabe 1 ist der Kontext, lassen wir Schnecke und Schwalbe einmal beiseite, jedoch ein anderer. Im zweiten Teil der Aufgabe geht es nämlich um die Ermittlung von Zeit, in Gestalt einer Umrechnung des Wertes von 90.000 Tagen in die konventionelle Einheit des Jahres. Tatsächlich lassen sich gerade im Umfeld der Komputistik viele solcher Umrechenaufgaben finden; ein schönes Beispiel bildet der Kalender aus einer Handschrift der Kölner Dombibliothek 83-2 aus dem Jahr 805, der mit folgender Passage

32. Propositio 1 (ed. M. FolKeRTS, S. 45) : Limax fuit ab hirundine invitatus ad prandium infra leuvam unam. In die autem non potuit plus quam unam unciam pedis ambulare. Dicat, qui velit, in quot diebus adidem prandium ipse limax perambulaverit. Solutio. In leuva una sunt mille quingenti passus, VIID pedes, XC unciae. Quot unciae, tot dies fuerunt, qui faciunt annos CCXLVI et dies CCX.

33. Beda, De temporum ratione, cap. 4 (ed. Ch. JonEs, S. 184-186).

34. Abbildung aus: Beda, De temporum ratione, ed. Ch. Jones, Kap. 4, S. 184-185. 
endet: „Ein Jahr enthält 12 Monate, 52 Wochen und eine Tag und 6 Stunden, 8760 (richtig 8766) Stunden und 87600 Momenta $^{35}$." Auch im Werk De computo des Hrabanus Maurus finden sich in allen Kapiteln zu den zeitlichen Bestimmungsgrößen wie Tag, Monat oder Jahr immer wieder solche Reihen einzelner komputistischer Parameter, die rechnerisch miteinander in Beziehung gesetzt werden ${ }^{36}$.

Auch hier sehen wir folglich, wenn auch in eine ganz andere Rahmenhandlung eingekleidet, eine Aufgabenstellung, die direkte Parallelen zur Komputistik aufweist. Angesichts des bereits angesprochenen Bedeutungsgehaltes der Osterfestberechnung in der Karolingerzeit kann daher auf ein konkretes Anforderungspotential auf diesem Sektor geschlossen werden, auf das Alkuin hier reagiert.

Daneben gibt es aber auch noch Aufgaben, deren Kontext möglicherweise auf deutlich profanere Zusammenhänge abzielt. So wird in Aufgabe 37 geschildert ${ }^{37}$, dass 6 Handwerker, nämlich fünf Meister und ein Lehrling, ein Haus errichten und dafür zusammen 25 Denare pro Tag als Lohn erhalten, und zwar derart, dass der Lehrling die Hälfte von dem erhält, was ein Meister bekommt. Die Aufgabe führt zu dem Problem, durch 11 teilen zu müssen, was in der Unzenrechnung unmöglich ist. Alkuin bot, demgemäß in Kenntnis der Schwierigkeiten, eine alternative Lösung an. Er ermittelt zunächst das Ergebnis der ganzzahligen Teilung, indem er 22 Denare aufteilt. Danach werden die restlichen 3 Denare in 33 Teile zerlegt, wodurch er eine den ganzen Denaren entsprechende Verteilung erreicht. Dabei könnte das geforderte Teilen des Denars, wie eingangs erwähnt, durchaus wörtlich verstanden werden. Alkuin gliedert die Rechnung also in Einzelschritte, die mit den Methoden des ganzzahligen Rechnens zu lösen sind; die disfunktionale Unzenrechnung wird nicht angesprochen (Abb. 2). Stattdessen führt er - möglicherweise in Reaktion auf die gängige Praxis in Wirtschaft und Handel - die Aufgabe auf das Gruppieren von Einheiten zurück. Wir müssen folglich davon ausgehen, dass das hier angebotene Lösungsspektrum durchaus vielfältig ist, und auf Anforderungen ver-

35. B. ENGLISCH, Zeiterfassung und Kalenderprogrammatik ..., S. $108:$ ANNI $\bullet$ MENSES • XII $\bullet$ EBDOMADAS • LII • ET UNA DIES • CCCLXV • DIES ET VI HORE •VIIIIDCCLX (HORE •) MOMENTA CCCLDC(C)•LX.

36. Hrabanus Maurus, De computo, S.163-331, hier z. B. cap. 27, S. 231 oder cap. 32, S. 236-240.

37. Propositio 37 (ed. M. Folkerts, S. 67) : Homo quidam volens aedificare domum locavit artifices VI, ex quibus V magistri et unus discipulus erat. Et convenit inter eum, qui aedificare volebat, et artifices, ut per singulos dies XXV denarii eis in mercede darentur, sic tamen, ut discipulus medietatem de eo, quod unus ex magistris accipiebat, acciperet. Dicat, qui potest, quantum unusquisque de illis per unumquemque diem accepit. Solutio. Tolle primum XXII denarios et divide eos in Vi partes. Sic da unicuique de magistris, qui quinque sunt, IIII denarios. Nam quinquies quattuor XX sunt. Duos, qui remanserunt, quae est medietas de IIII, tolle et da discipulo. Et sunt adhuc III denarii residui, quos sic distribues : Fac de unoquoque denario partes XI. Ter undecim fiunt XXXIII. Tolle illas triginta partes, divide eas inter magistros V. Quinquies seni fiunt XXX. Accidunt ergo unicuique magistro partes VI. Tolle tres partes, quae super $X X X$ remanserunt, quod est medietas senarii, ac dabis discipulo. 
schiedener Sachzusammenhänge und konkreter Situationen antwortet, in denen gerechnet werden musste. Auch dies würde aber bedeuten, dass die Propositiones eine wichtige, vielleicht die wichtigste Quelle über das Rechnen in der Karolingerzeit darstellen.

Zusammenfassend betrachtet scheint es sinnvoll zu sein, die Alkuin zugeschriebenen Propositiones im Kontext der Anforderungen zu betrachten, die die operative Arithmetik im Frühmittelalter bereitstellte. War es auch nur möglich, diese hier in Auswahl zu betrachten, so machte doch bereits diese kurze Quellenanalyse deutlich, dass es unzureichend ist, die dort vorgeführten Aufgaben lediglich auf eine intellektuelle Gedankenspielerei ohne Rückwirkung auf die tatsächlichen Fragestellungen dieser Epoche im Bereich des Quadriviums zu reduzieren. Vielmehr wurde deutlich, dass ein Großteil der Propositiones auf ein akutes Problem des praktischen Rechnens antwortet, nämlich die Unmöglichkeit, im System der römischen Zahlen eine Division durchzuführen. Damit gelingt Alkuin folglich nicht nur eine das Quadrivium, sondern das Bildungsgefüge selbst betreffende Innovation, nämlich die Abfassung einer Lehrschrift operativer Arithmetik, die die Enzyklopädien der Zeit sowohl qualitativ als auch gattungsspezifisch bei weitem überschreitet. Die Geltung dieser Überlegung sollte nicht auf die hier fokussierte Osterfestrechnung beschränkt bleiben; eingedenk des Faktums, dass auch in anderen Komplexen die Notwendigkeit zur Bewältigung solcher Rechenoperationen bestand, sollte dieser Ansatz ebenfalls auf die in den Propositiones erwähnten Sachbereiche Handel oder Klosterwirtschaft ausgeweitet werden. Vielleicht ist dann der Nachweis einer Leistung Alkuins möglich, die hier nur als These formuliert werden kann, dass ihm etwas gelungen ist, woran auch heute noch viele Autoren scheitern: ein auf konkrete mathematische Fragen seiner Zeit antwortendes, qualitativ hochwertiges und zugleich unterhaltsames Lehrbuch zu verfassen. 
Abb. 1 - Die Unzen nach Beda Venerabilis, De temporum ratione, cap. 4

$x$ libra uel as siue assis

is deunx siue jabus

ifr decunx uel dextans

sir dodrans siue doras

$u$ bes siue bisse

4 septunx siue septus

$s$ semis

Hr quincunx siue cingus

$\psi$ s triens siue treas

Y quadrans siue quadras

$\zeta$ sextans sive sextas

$y$ sescunx siue sescuncia

uncia

2 semuncia

$<$ duae sextulae siue sesclae, id est tertia pars unciae

7 sicilicus

L sextula siue sesclae

$\checkmark$ dimidia sextula siue sesclae

ff seripulus xii. unciae

-xi. unciae

'x. uneiae

viiii. unciae

-viii. unciae

-vii. unciae

.vi. unciae

$\cdot v \cdot$ unciae

iiii. uncize

.iii. unciae

.ii. unciae

i. uncis et semis

-xxiiii-ff scripuli

xii- scripuls

-viii. scripuli

vi- scripuli

.iiii. scripuli

.ii. scripuli

.vi. siliquae

Abb. 2 - Der Rechenweg zu Aufgabe 37

\section{Aufgabe 37}

\section{1) Verteilung der 25 ganzen Denare}

Voraussetzung:

5 Meister bekommen $2 \mathrm{x}$; der Lehrling $1 \mathrm{x}$

d. h. Alkuin rechnet

$$
\begin{aligned}
& 10 x+1 x=11 x \\
& 25: 11=2 \text { Rest 3 }
\end{aligned}
$$

Damit bekommt

jeder Meister 4 Denare

der Lehrling 2 Denare.

\section{2) Verteilung des Restes von 3 Denaren}

Voraussetzung:

Zerlegung der Denare in 33 Teile, d.h. es werden $33 / 11$ Denare verteilt Dies ergibt
jeweils 6 Teile $(5 \times 6=30)$
für die Meister, d. h. $\quad 6 / 11$ Denare
3 Teile für den Lehrling, d. h. 3/11 Denare (also erneut die Hälfte)
Jeder Meister erhält folglich $4 \%$ Denare und der Lehrling $\quad 2 \frac{3}{11}$ Denare 


\section{ZUSAMMENFASSUNG}

Alkuins populärstes Werk zu den Themenbereichen des Quadriviums sind die ihm zugeschriebenen Propositiones ad acuendos juvenes, die bedeutendste frühmittelalterliche Sammlung von Rechen - und Scherzaufgaben in lateinischer Sprache. Es ist jedoch nicht ausreichend, sie als Unterhaltungsmathematik gewissermaßen allein als intellektuelle Gedankenspielerei zu betrachten. Ein großer Teil der Propositiones thematisiert Fragen der operativen Arithmetik im Kontext der Komputistik, die Klosterwirtschaft, Handwerk und Handel im Frühmittelalter prägten. Mehr als die Hälfte der Aufgaben antwortet auf ein konkretes Problem des praktischen Rechnens, nämlich die Unmöglichkeit, im System der römischen Zahlen eine Division durchzuführen. Mit der Abfassung dieser Lehrschrift zur operativen Arithmetik gelingt Alkuin folglich eine nicht nur das Quadrivium, sondern das Bildungsgefüge selbst betreffende Innovation, welche die Enzyklopädien der Zeit sowohl qualitativ als auch gattungsspezifisch überschreitet. Die Propositiones sollten daher als ein auf konkrete mathematische Fragen ihrer Zeit antwortendes, qualitativ hochwertiges und zugleich unterhaltsames Lehrbuch bewertet werden.

\section{ABSTRACT}

Alkuins most popular work attributed to him, regarding to the fields of the Quadrivium, are the Propositions ad acuendos juvenes. It is the most important early medieval collection of calculation problems and mathematical riddles in Latin. It is, however, not sufficient to consider this collection only as "Unterhaltungsmathematik", simply as intellectual pastime basing on arithmetics so to speak. For the most part Propositiones deals with concrete arithmetical issues in the context of the medieval calculation of Easter, the computus, of the economy of a medieval monastery or of craft and trade during the Early Middle Ages. More than half of the issues give answers to a concrete problem of practical calculation, namely, the impossibility to accomplish a division in the system of the Roman numbers. With the composition of this proposition to applied arithmetics, Alkuin succeeded in having innovated not only the Quadrivium, but also the medieval education system itself. This innovation oversteps the encyclopaediae of those times both qualitatively as well as specific to this genre. Propositiones should thus be rated as a qualitatively high - graded and at the same time entertaining textbook, which gives answers to concrete mathematical issues of its time. 